\title{
A novel Extended Impedance-Power droop for accurate active and reactive power sharing in a multi-bus microgrid with complex impedances
}

\author{
Reza Razi, Hossein Iman-Eini*, Senior Member, IEEE, Mohsen Hamzeh, Member, IEEE, \\ Seddik Bacha, Senior Member, IEEE
}

\begin{abstract}
This paper addresses a novel and flexible control algorithm based on complex virtual impedance for improvement of power sharing in a multi-bus microgrid with complex impedance of lines. In the proposed method, first, the Extended Impedance-Power droop equations are derived. Using nonlinear droop equations, equivalent resistance and inductance of each DG unit are obtained by active and reactive power, separately. Then, an appropriate algorithm is employed to implement ImpedancePower droop to manage the virtual impedances adaptively. The proposed algorithm can optimally share both active and reactive power, even when the structure is changed without any knowledge of microgrid parameters and high-bandwidth communication links. The parameters of the proposed controller are designed based on the nominal power of inverters. Moreover, the stability margin of the system is analyzed. Finally, the feasibility of the proposed control scheme is confirmed via simulations and experiments.
\end{abstract}

Index Terms-Complex impedance, impedance-power droop, multi-bus microgrid, power sharing, virtual impedance.

\section{INTRODUCTION}

$\mathrm{I}_{\mathrm{s} e}^{\mathrm{N}}$ $\mathrm{N}$ recent years, extensive researches on renewable energy sources (RES) and distributed generation (DG) has taken place as a promising solution to compensate energy shortages and environmental problems [1], [2]. DG units typically connect to the microgrid with parallel inverters serving as interface devices. The structure of parallel inverters can be mainly divided into two categories: the single-bus type and a multi-bus one [3]. In the single-bus structure, all inverters are connected to the common ac bus through the respective feeders. Nevertheless, in the multi-bus structure, there is no common bus and inverters can be connected freely to any point of a microgrid. The multi-bus structure is generic and includes the single-bus structure.

As the number of DG units increases, there are some notable issues, such as power management, synchronization, resonance, and system stability [4]. In this regard, the parallel operation of

This work has been supported in part by the m2M-GRID project of the joint programming initiative ERA-Net Smart Grids Plus.

R. Razi, H. Iman-Eini and M. Hamzeh are with the School of Electrical and Computer Engineering, College of Engineering, University of Tehran, Iran (email: reza.razi@ut.ac.ir, imaneini@ut.ac.ir,mohsenhamzeh@ut.ac.ir).

R. Razi and S.Bacha are with Univ. Grenoble Alpes, CNRS, Grenoble INP, G2Elab, Grenoble, France (e-mail: reza.razi@g2elab.grenoble-inp.fr, seddik.bacha@g2elab.grenoble-inp.fr). inverters is pursued as a key technique in the control system. In fact, load power demands must be shared in proportion to the nominal power of islanded parallel inverters in order to avoid overload, power quality issues, additional stress and delay aging [5]-[7].

The droop method is the most popular control method for power sharing requirement in parallel inverters [8]. The conventional droop method works well in a single-bus microgrid with inductive line impedances. Besides, in a low voltage microgrid, where the feeder impedance is resistive, it is better to use the $\mathrm{P} / \mathrm{V}$ and $\mathrm{Q} / \mathrm{f}$ droop characteristics. As stated in the literature, this method has some known limitations regarding power quality [9]. To overcome this drawback, various improved methods have been presented based on droop's characteristics. For example, in [10], the optimal angle droop control method is introduced that uses phase angle for power sharing enhancement with improvement in stability. The main advantage of this method is the low-frequency deviation, but the asynchronous operation of the controllers can lead to a non-identical frequency in inverters and even instability. Furthermore, in [11], [12], voltage regulation, stability and power sharing have been improved with the adaptive droop control method, but physical parameters must be identified. In [13], a developed double-droop voltage controller is proposed that the overall system stops when a communication error occurs. In addition, the mentioned methods use R-type or Ltype droop, which may lead to an unstable mode in microgrids with complex impedance of lines due to the coupling between active and reactive power.

The virtual impedance technique is another improved method in droop control that is introduced to solve the problems of system control instability and inaccurate power sharing, simultaneously [14]. In this technique, the output voltage reference is improved by a current feedforward loop, but due to the mismatch of large equivalent impedances, the powersharing error is not completely eliminated, and the quality of output voltage will get worse. In [15] and [16], equivalent impedances are calculated using the output current and feeder impedance, and these problems are solved with the help of adaptive adjustable virtual impedance. However, it is difficult to identify the feeder impedance in practical applications and the proposed method will be ineffective. Moreover, in [17], a simple impedance-power droop method is proposed for the 
parallel inverters but only in islanded resistive low voltage microgrids. Until here, the mentioned methods focus on the single-bus structure. In fact, previous studies in relation to single-bus structures cannot be generalized to multi-bus structures. In the multi-bus structure, the feeder impedance cannot be determined for each inverter. In addition, this structure is not constant and the impedance between two nodes can change continuously.

In [18], a virtual impedance controller is presented based on the exact recognition of the microgrid structure to eliminate the power-sharing error in a multi-bus structure. This method is no longer valid when the microgrid structure changes. In [19] and [20], consensus-based controllers are used to adjust the virtual impedances adaptively, which is very complicated due to a significant number of controller gains. Also, the virtual impedance obtained in the consensus protocol is large, which causes power quality problems. In [3], the power-sharing performance is improved in a multi-bus structure with virtual resistors, but the robustness of the controller is not guaranteed when changing the local load or microgrid structure. Recently, the use of low-bandwidth communication (LBC) and central controller have been proposed in [21] to improve the control plan. However, this method cannot take into account the restructuring of the microgrid and the system reliability is reduced by using the central controller. Therefore, most of the existing methods in the literature are based on system information that is not usually available or identifiable, such as line impedance and equivalent load.

Motivated by the aforementioned challenges, in this paper, a novel control strategy based on virtual impedance is presented, called Impedance-Power droop, which can effectively ensure the power sharing in a multi-bus microgrid with complex impedance of lines. The proposed method can be easily implemented in any type of microgrid topology without the need for network structure information and high-bandwidth communication. It also works properly by changing the microgrid structure or load conditions. Significant features of the proposed method are:

- Considering coupling between active and reactive powers,

- No frequency deviation caused by load control,

- Voltage control without the need for a central controller.

\section{POWER SHARING THEORETICAL BACKGROUND}

In this section, the effective factors in injected power by each inverter are examined. An islanded inverter can be considered as an ideal voltage source and an inherent series impedance [22]. Therefore, the parallel connection of the inverters thorough feeder impedances in the single-bus structure is modeled as Fig. 1. $Z_{L_{i}}, Z_{\text {inv }_{i}}$ and $Z_{t_{i}}$ represent the feeder impedance, inherent inverter impedance and total impedance for inverter $i$, respectively. $V_{r e f_{i}}, I_{i}$ and $V_{o}$ are the reference voltage, the output current of the inverter and the common bus voltage for inverter $i$, respectively.

The apparent power injection into the microgrid by each inverter can be expressed as,

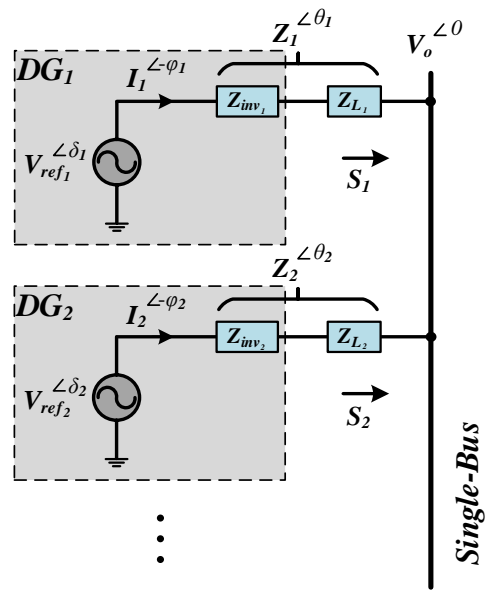

Fig. 1. Single-bus structure of islanded parallel inverters.

TABLE I

DIFFERENT TYPES OF POWER EQUATIONS

\begin{tabular}{|c|c|c|}
\hline Type & Impedance angle & Power equations \\
\hline $\mathrm{L}$ & $\Theta=90^{\circ}$ & $P_{i}=\frac{V_{r e f_{i}} V_{o} \sin \delta_{i}}{X_{i n v_{i}}+X_{L_{i}}}, Q_{i}=\frac{V_{r e f_{i}} V_{o} \cos \delta_{i}-V_{o}^{2}}{X_{i n v_{i}}+X_{L_{i}}}$ \\
\hline RL & $0^{\circ}<\Theta<90^{\circ}$ & $P_{i}=\frac{V_{r e f_{i}} V_{o} \cos \left(\phi_{i}-\delta_{i}\right)-V_{o}^{2} \cos \phi_{i}}{Z_{i n v_{i}}+Z_{L_{i}}}, Q_{i}=\frac{V_{r e f_{i}} V_{o} \sin \left(\phi_{i}-\delta_{i}\right)-V_{o}^{2} \sin \phi_{i}}{Z_{i n v_{i}}+Z_{L_{i}}}$ \\
\hline $\mathrm{R}$ & $0^{\circ}$ & $P_{i}=\frac{V_{r e f_{i}} V_{o} \cos \delta_{i}-V_{o}^{2}}{R_{i n v_{i}}+R_{L_{i}}}, Q_{i}=-\frac{V_{r e f_{i}} V_{o} \sin \delta_{i}}{R_{\text {invi }}+R_{L_{i}}}$ \\
\hline $\mathrm{RC}$ & $-90^{\circ}<\Theta<0^{\circ}$ & $P_{i}=\frac{V_{r e f_{i}} V_{o} \cos \left(\phi_{i}-\delta_{i}\right)-V_{o}^{2} \cos \phi_{i}}{Z_{i n v_{i}}+Z_{L_{i}}}, Q_{i}=\frac{V_{r e f_{i}} V_{o} \sin \left(\phi_{i}-\delta_{i}\right)-V_{o}^{2} \sin \phi_{i}}{Z_{i n v_{i}}+Z_{L_{i}}}$ \\
\hline $\mathrm{C}$ & $\Theta=-90^{\circ}$ & $P_{i}=-\frac{V_{r e f_{i}} V_{o} \sin \delta_{i}}{X_{i n v_{i}}+X_{L_{i}}}, Q_{i}=-\frac{V_{r e f_{i}} V_{o} \cos \delta_{i}-V_{o}^{2}}{X_{i n v_{i}}+X_{L_{i}}}$ \\
\hline
\end{tabular}

$S_{i}=V_{r e f_{i}} \times I_{i}^{*}=V_{r e f_{i}} e^{j \delta_{i}}\left(\frac{V_{r e f_{i}} e^{-j \delta_{i}}-V_{o}}{Z_{t_{i}} e^{-j \theta_{i}}}\right)$

$=\frac{V_{r e f_{i}}{ }^{2}}{Z_{t_{i}}} e^{j \theta_{i}}-\frac{V_{r e f} V_{o}}{Z_{t_{i}}} e^{j\left(\theta_{i}+\delta_{i}\right)}$

As shown in Equation (1), the apparent power of each inverter is related to the reference voltage $\left(V_{\text {ref }}\right)$ and total impedance $\left(Z_{t}\right)$. In fact, with the assumption of two DG units, the load current $I_{L}$ is divided according to (2), where a DG unit with a lower rate may supply more power.

$I_{1}=\frac{Z_{t_{2}}}{Z_{t_{1}}+Z_{t_{2}}} I_{L}+\frac{V_{r e f_{1}}-V_{\text {ref }}}{Z_{t_{1}}+Z_{t_{2}}}, I_{2}=\frac{Z_{t_{1}}}{Z_{t_{1}}+Z_{t_{2}}} I_{L}+\frac{V_{\text {ref }}-V_{\text {ref }}}{Z_{t_{1}}+Z_{t_{2}}}$

In power sharing control methods based on droop's characteristics, the relationship between power and reference voltage is considered. However, the droop equations are obtained in accordance with the characteristic of the equivalent impedance and assuming a small power angle $(\delta)$. Table I shows different types of power equations based on the equivalent output impedance. In this paper, another variable dependent on apparent power, i.e. total impedance, is used to control injectable power. In other words, by providing a novel control method based on complex virtual impedance, accurate power sharing is obtained in a multi-bus structure with complex impedances. In order to provide a simple expression and better understanding, the proposed method is introduced in the singlebus structure and then generalized to the multi-bus one. 


\section{PROPOSED CONTROL STRATEGY}

As stated in [23], using the virtual impedance technique to improve power sharing in multi-bus microgrid requires some information such as feeder impedances, microgrid structure and local and common loads power. This information is timevariable, it is not readily available and it increases the computational burden. In this paper, the virtual impedance technique is used in another way to solve these problems. In fact, the proposed strategy attempts to eliminate some uncertainties and to use parameters that are easily accessible or detectable.

Derived from (1), the injected apparent power is inversely proportional to the total impedance module. As a result, it is possible to control the apparent power by changing the inverter's effective impedance. In literature, the most common way to change the effective impedance is to use the virtual impedance technique according to Equation (3),

$V_{\text {ref }}^{\text {new }}=V_{\text {ref }}^{\text {old }}-I_{L_{i}} \cdot Z_{v_{i}}$

where $V_{\text {ref }}^{\text {new }}$ is the new reference voltage, $V_{\text {ref }}^{\text {old }}$ is the old reference voltage and $Z_{v}$ is virtual impedance. Therefore, to increase (decrease) the apparent power of the inverter, negative (positive) virtual impedance can be used. As a result, inspired by the traditional droop equations, a relationship between apparent power and virtual impedance, called ImpedancePower droop, can be obtained.

$$
\left(Z_{v}\right)^{\text {new }}=\left(Z_{v}\right)-b \times S
$$

where $b$ is the droop coefficient. Impedance-power droop method is well described in [17].

Equation (4) uses virtual impedance for apparent power sharing. If the resistive or inductive part of the equivalent impedance is dominant enough, active and reactive power are automatically shared and decoupled. Nevertheless, in a microgrid with complex impedances, the apparent power sharing cannot be generalized to active and reactive power. So, the impedance-power droop can be provided for a complex microgrid.

\section{A. Extended Impedance-Power droop}

From (1), the following relationships can be obtained for active and reactive powers:

$\left\{\begin{array}{l}P_{i}=\frac{V_{r^{2 e f_{i}}}^{2}}{Z_{t_{i}}} \cos \left(\theta_{i}\right)-\frac{V_{r e f_{i}} V_{o}}{Z_{t_{i}}} \cos \left(\theta_{i}+\delta_{i}\right) \\ Q_{i}=\frac{V_{r e f_{i}}^{2}}{Z_{t_{i}}} \sin \left(\theta_{i}\right)-\frac{V_{r e f_{i}} V_{o}}{Z_{t_{i}}} \sin \left(\theta_{i}+\delta_{i}\right)\end{array}\right.$

with $Z_{t_{i}} e^{j \theta_{i}}=R_{t_{i}}+j X_{t_{i}}$, (5) is rewritten as

$\left\{\begin{array}{l}P_{i}=V_{\text {ref }_{i}}\left[\frac{R_{t_{i}}}{R_{t_{i}}{ }^{2}+X_{t_{i}}{ }^{2}}\left(V_{\text {ref }_{i}}-V_{o} \cos \left(\delta_{i}\right)\right)+\frac{X_{t_{i}}}{R_{t_{i}}{ }^{2}+X_{t_{i}}{ }^{2}} V_{o} \sin \left(\delta_{i}\right)\right] \\ Q_{i}=V_{\text {ref }_{i}}\left[\frac{-R_{t_{i}}}{R_{t_{i}}{ }^{2}+X_{t_{i}}{ }^{2}} V_{o} \sin \left(\delta_{i}\right)+\frac{X_{t_{i}}}{R_{t_{i}}{ }^{2}+X_{t_{i}}{ }^{2}}\left(V_{r e f_{i}}-V_{o} \cos \left(\delta_{i}\right)\right)\right]\end{array}\right.$

Hence, the relationship between power and impedance can be calculated as

$\left[\begin{array}{c}P_{i} \\ Q_{i}\end{array}\right]=V_{r e f_{i}}\left[\begin{array}{cc}t_{i} & s_{i} \\ -s_{i} & t_{i}\end{array}\right]\left[\begin{array}{c}\frac{R_{t_{i}}}{R_{t_{i}}^{2}+X_{t_{i}}^{2}} \\ \frac{X_{t_{i}}}{R_{t_{i}}^{2}+X_{t_{i}}^{2}}\end{array}\right]$

where,

$$
\begin{aligned}
& t_{i}=V_{\text {ref }_{i}}-V_{o} \cos \left(\delta_{i}\right) \\
& s_{i}=V_{o} \sin \left(\delta_{i}\right)
\end{aligned}
$$

By solving the matrix equation (7), the following

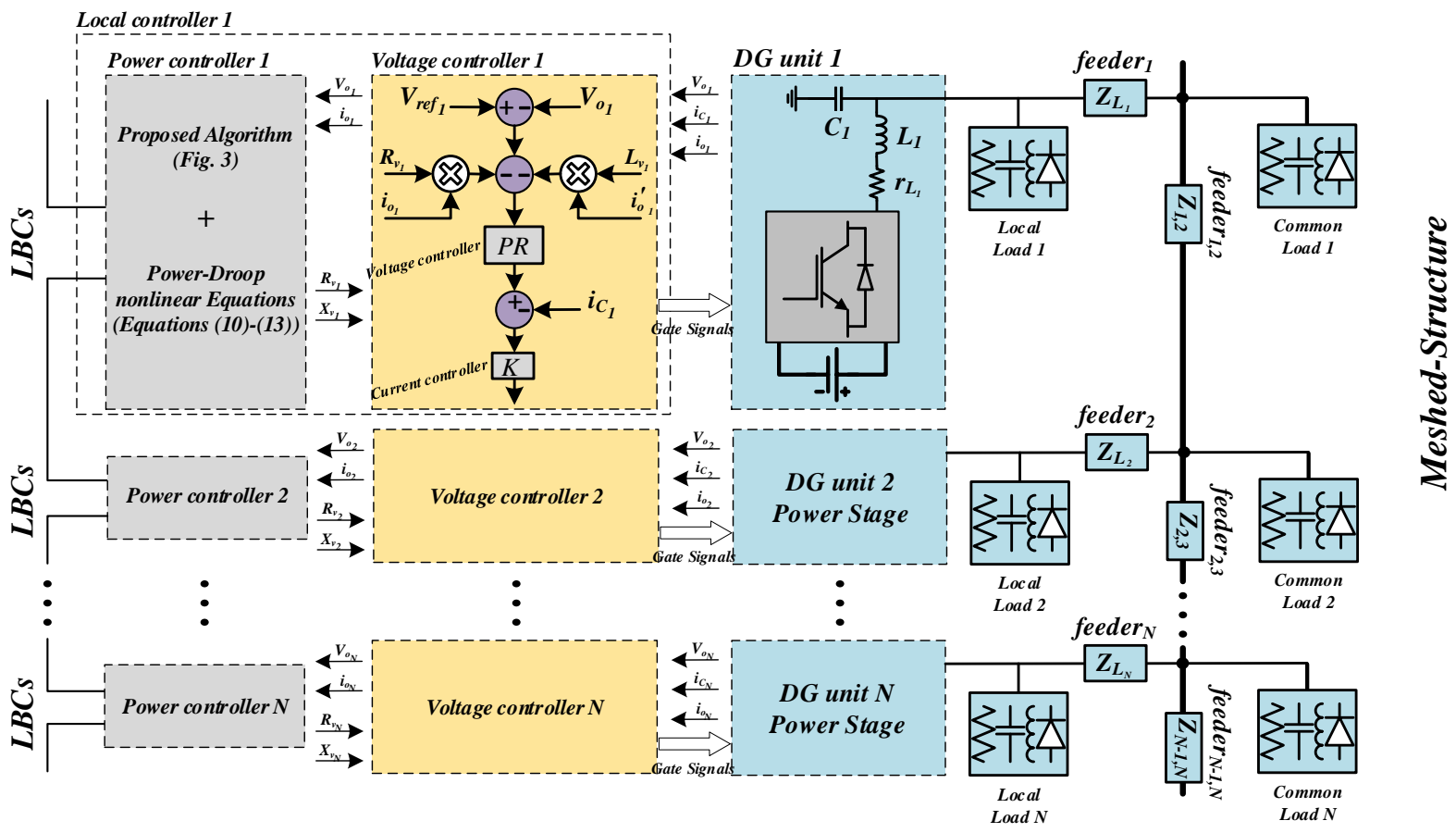

Fig. 2. Overall block diagram of the desired multi-bus microgrid. 
relationships are obtained

$$
\begin{aligned}
& R_{i}=\frac{V_{r e f_{i}}\left(t_{i}^{2}+s_{i}^{2}\right)\left(t_{i} \times P_{i}-s_{i} \times Q_{i}\right)}{\left(t_{i} \times P_{i}-s_{i} \times Q_{i}\right)^{2}+\left(s_{i} \times P_{i}+t_{i} \times Q_{i}\right)^{2}}=f_{r_{i}}\left(P_{i}, Q_{i}, v_{o}\right) \\
& X_{i}=\frac{V_{r e f_{i}}\left(t_{i}^{2}+s_{i}^{2}\right)\left(s_{i} \times P_{i}+t_{i} \times Q_{i}\right)}{\left(t_{i} \times P_{i}-s_{i} \times Q_{i}\right)^{2}+\left(t_{i} \times P_{i}-s_{i} \times Q_{i}\right)^{2}}=f_{x_{i}}\left(P_{i}, Q_{i}, v_{o}\right)
\end{aligned}
$$

As can be seen in (10) and (11), equivalent resistance and inductance of each inverter are nonlinear functions of active power, reactive power, and bus voltage. Dependence on bus voltage is one of the drawbacks in control methods [16], [18], but this paper overcomes this problem using the singular characteristics of the extended impedance-power droop method. In fact, in this method, the output voltage can be approximated as a constant variable. Similar to (4), the nonlinear equations of extended Impedance-Power droop are defined as follows

$$
\begin{aligned}
& R_{v_{i}}^{\text {new }}=R_{v_{i}}-\left(f_{r_{i}}\left(P_{i}\left(b_{r_{i}}\right), Q_{i}\left(b_{r_{i}}\right)\right)-f_{r_{i}}\left(P_{i}, Q_{i}\right)\right) \\
& X_{v_{i}}^{\text {new }}=X_{v_{i}}-\left(f_{x_{i}}\left(P_{i}\left(b_{x_{i}}\right), Q_{i}\left(b_{x_{i}}\right)\right)-f_{x_{i}}\left(P_{i}, Q_{i}\right)\right)
\end{aligned}
$$

where $b_{r}$ and $b_{\mathrm{x}}$ are the droop coefficients, and $R_{v}$ and $X_{v}$ are the virtual resistive and virtual inductive, respectively. By using these equations in the structure of an appropriate algorithm in the next section, the values for virtual resistance and inductance can be selected so that exact power sharing is obtained.

\section{B. Proposed algorithm to implement droop equations}

In the previous section, the relation of equivalent resistance and inductance was obtained in terms of other system parameters. Here, with the help of these relationships and the proposed algorithm, the values of virtual resistance and inductance are adaptively obtained to achieve the desired output power.

Various methods have been introduced in the literature to control islanded single inverters [24]. In this paper, the multiloop control in [25], is inspired. In this control structure, the PR controller is used in the main loop to adjust the output voltage of the inverter and a simple proportional controller is used in the inner loop to compensate for disturbances and increase the system dynamics. The overall block diagram of the desired multi-bus microgrid is shown in Fig. 2. System parameters are also listed in Table II.

As shown in Fig. 2, the values of virtual resistance and inductance are transmitted to the voltage controller from the power controller. In this paper, the algorithm, which is shown in Fig. 3, is used to calculate complex virtual impedances. In general, this algorithm consists of four parts: 1 . The output fundamental power of each inverter is calculated locally using the voltage and output current. 2 . The power is multiplied by the droop gain and pulse train and sent to related DGs. The purpose of applying droop gain is to obtain a benchmark for comparing the power of inverters. In addition, the pulse train is used to create a duty cycle in the proposed algorithm. 3 . The power changes on each inverter are calculated using local data and received information from other units (one unit is enough). 4. The final power for each inverter is specified and the virtual resistance and inductance are updated using extended droop equations.
TABLE II

SYSTEM PARAMETERS

\begin{tabular}{|c|c|c|}
\hline \multicolumn{3}{|c|}{ SYSTEM PARAMETERS } \\
\hline LC filter & $L / r_{L} / C$ & $2 \mathrm{mH} / 0.2 \Omega / 20 \mu \mathrm{f}$ \\
\hline DC link voltage & $V_{D C}$ & $400 \mathrm{~V}(\mathrm{sim})$. \\
\hline Operating voltage & $v_{o}$ & $200 \mathrm{~V}(\mathrm{exp})$. \\
\hline Switching frequency & $f_{s}$ & $70 \mathrm{~V}, 50 \mathrm{~Hz}(\mathrm{exp})$. \\
\hline Control parameters & Symbol & $20 \mathrm{kHz}$ \\
\hline PR controller gains & $k_{p} / k_{i}$ & Value \\
\hline Proportional controller & $k$ & $0.125 / 15$ \\
\hline Cutoff frequency & $\omega_{c}$ & 36.2 \\
\hline Network parameters & Symbol & $5 \mathrm{rad} / \mathrm{s}$ \\
\hline DG feeder & $Z_{L 1} / Z_{L 2}$ & $0.5 \Omega, 2 \mathrm{mH} / 1 \Omega, 1 \mathrm{mH}(\mathrm{exp})$. \\
\hline Network feeder & $Z_{l, 2}$ & $0.5 \Omega, 0.2 \mathrm{mH}(\mathrm{sim})$. \\
\hline
\end{tabular}

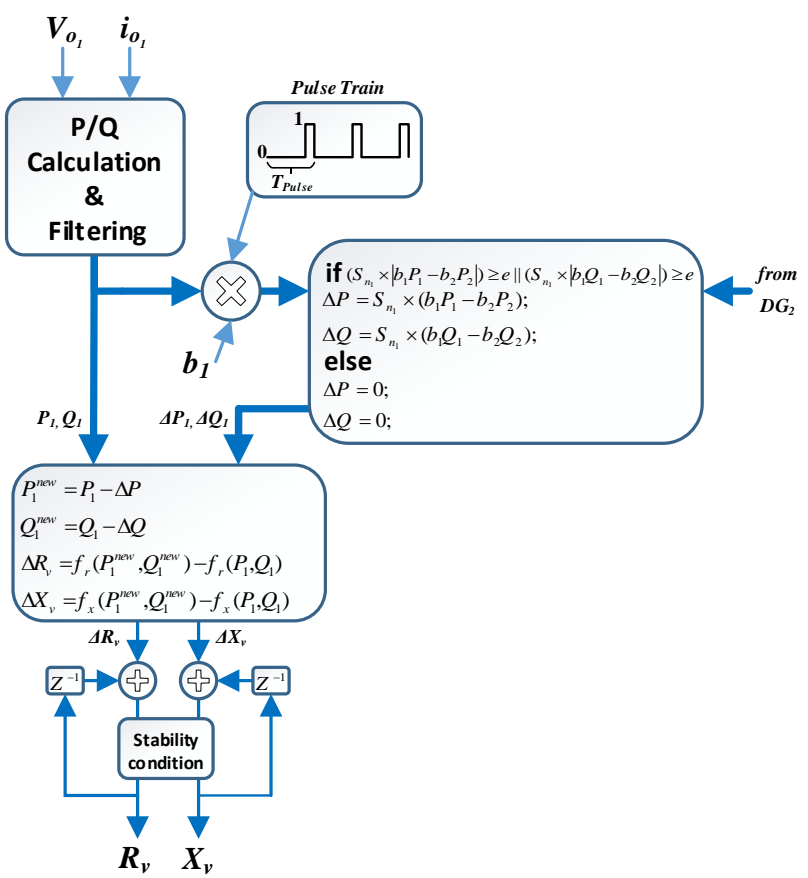

Fig. 3. Proposed algorithm to implement droop equations.

Regarding the algorithm structure, the droop equations are applied to the system at any period $\left(T_{\text {pulse }}\right)$. In the case of a small duty cycle, the algorithm does not have enough time to change the power of inverters with new virtual impedances. Here, the period is assumed 20 milliseconds.

The parameter $b$ plays two roles in the extended impedancepower algorithm: 1 . Power normalization (denominator of the parameter) 2. Step-by-step compensation of the power difference (numerator of the parameter). In the first role, the output power of the inverters is calculated relative to their nominal power to obtain a suitable criterion for comparison. In the second role, the step of compensating the power difference is determined. This role is defined by the tradeoff between the accuracy and speed of the algorithm. If a small b is chosen, the less power difference is compensated in each period of the algorithm, but the more accurate power-sharing condition can be applied. In contrast, by choosing a larger value for $b$, although the power difference compensation speed increases, 
the accuracy of power-sharing decreases. In this paper, $b$ is determined in such a way that at each run of the algorithm, 10\% of the power difference between the inverters is compensated.

$b_{i}=0.1 / S_{n_{i}}$

It should be noted that in this work, it is assumed that the power factors of the inverters are similar; in this case, $b$ is independent of the power factor. Obviously, assuming different power factors for parallel inverters, $b$ is not the same for active and reactive power and is modified as

$b_{P}=\frac{0.1}{P_{n}}=\frac{0.1}{S_{n} \times \cos \phi}$

$b_{Q}=\frac{0.1}{Q_{n}}=\frac{0.1}{S_{n} \times \sin \phi}$

With respect to the definition of $b,(17)$ is used to verify the power sharing.

$S_{n_{1}} \times\left(b_{1} P_{1}-b_{2} P_{2}\right) \geq e_{p}$ or $S_{n_{1}} \times\left(b_{1} Q_{1}-b_{2} Q_{2}\right) \geq e_{q}$

where $e$ shows the acceptable error of the power difference. In fact, the algorithm continues as long as this equation is satisfied. $e_{p}$ and $e_{q}$ are considered to be $0.05\left(P_{1}+P_{2}\right)$ and $0.05\left(Q_{1}+Q_{2}\right)$, respectively, in which power sharing is accepted by a margin of lower than $10 \%$.

Obviously, this algorithm is inherently resistant against the delay of communication links because it has enough time to send information, as shown in Fig. 4 . In fact, in each $T_{\text {pulse }}$, the parameters must be updated and the link delay is not affected. In addition, if the communication link is disconnected, the virtual impedance will remain in its final value, and as long as the network does not change, there is no need for a link.

\section{Stability and convergence speed analysis}

It should be noted that acting on impedance could have a serious effect on system stability. To investigate the effect of the virtual impedance obtained from extended droop equations on system stability, the Routh-Hurwitz stability criterion is used. For each converter, the transfer function of the inner current control loop is obtained as

$G_{i}(s)=\frac{i_{C}}{i_{C, \text { ref }}}=\frac{Z C k s}{Z C L s^{2}+\left(Z C\left(r_{L}+k\right)+L\right) s+r_{L}}$

where $Z$ is the load impedance. The phase margin (PM) and closed-loop stability are reduced slightly under no-load or lightload conditions [25]. Therefore, the stability study is conducted under these conditions (worst-case), although it simplifies the analysis, but confirms the stability for all operating conditions. Given the converter at light-load $(Z=>\infty)$, Equation (18) is changed as

$G_{i}(s)=\frac{i_{C}}{i_{C, r e f}}=\frac{k}{L s+r_{L}+k}$

Finally, the characteristic equation of the voltage control system, in which the virtual impedance resulting from the extended impedance-power algorithm is also considered, can be written as [17]

$1+\left(1+\frac{Z_{v}}{Z}\right) G_{P R}(s) G_{i}(s) \frac{1}{C s}=0$

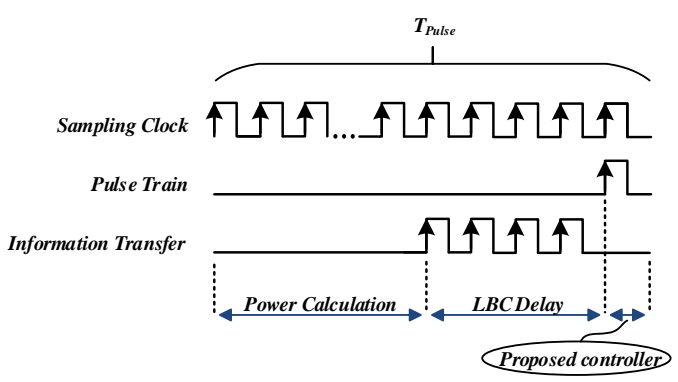

Fig. 4. Control timing diagram in a $T_{p u l s e}$.

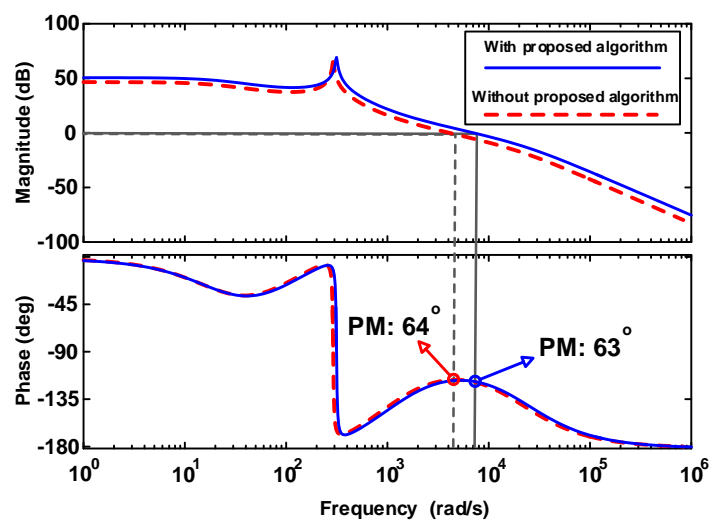

Fig. 5. Analysis of PM with and without proposed algorithm.

Assuming $R_{v} \ll Z$ and $L_{v} \ll Z / 10^{3}$, the characteristic equation is simplified as

$$
\begin{aligned}
& 4 \times 10^{-8} s^{4}+\left(7.284 \times 10^{-4}+\frac{L_{v}}{Z} 4.525\right) s^{3}+4.525 s^{2}+5475 s \\
& +4.461 \times 10^{5}=0
\end{aligned}
$$

By applying the Routh-Hurwitz stability criterion to (21), the stability conditions of the system are obtained as

$L_{v}>-0.161 \times 10^{-3} \times Z$

$L_{v}<12.112 \times 10^{-3} \times Z$

As can be seen, the virtual resistor does not affect the stability of the system but the virtual inductance is limited to the upper and lower bands in terms of load impedance. In our case, the load impedance in the worst-case conditions equals $20 \mathrm{ohms}$, which results in

$-3.22 \times 10^{-3}<L_{v}<242.24 \times 10^{-3}$

Equation (24) shows that although the upper band is unavailable, lower band discriminant must be considered. Therefore, this equation is examined in the last step of the proposed algorithm, and if this is violated, the value of the virtual inductance returns to the nearest stable area value.

After checking the system stability, the stability margin is also evaluated. The bode plot of open-loop transfer function with and without the proposed algorithm is plotted in Fig. 5 [17]. As shown, the PM in the presence of virtual impedance in the worst-case conditions is reduced by only 1 degree, which can be ignored and PM is sufficient for power electronic applications.

Besides, the convergence speed analysis can provide a better view of the performance of the impedance-power method. It is very complicated in the microgrids with complex impedances 
to investigate the convergence speed of active and reactive power sharing, simultaneously. Nevertheless, it is assumed for simplicity that the microgrid is purely resistive or inductive that the active and reactive powers do not affect each other. In the first period of the power sharing compensation, according to Equation (14), each of the converters compensates 10 percent of the power difference. So at the end of this period, the power difference is calculated as

$\Delta P_{1}=\left|P_{1}-P_{2}\right|-\left|\frac{P_{1}-P_{2}}{10}\right|-\left|\frac{P_{1}-P_{2}}{10}\right|=0.8 \times\left|P_{1}-P_{2}\right|$

In the next period, each of the converters shall again compensate 10 percent of the power difference in the previous period as

$\Delta P_{2}=0.8 \times\left|P_{1}-P_{2}\right|-2 \times\left|\frac{0.8 \times\left(P_{1}-P_{2}\right)}{10}\right|=(0.8)^{2} \times\left|P_{1}-P_{2}\right|$

Finally, the power difference at the end of the $\mathrm{n}^{\text {th }}$ period is obtained from the simple following formula:

$\Delta P_{n}=(0.8)^{n} \times\left|P_{1}-P_{2}\right|$

Therefore, the impedance-power droop algorithm reduces the power difference to about $10 \%$ of the initial power difference after 10 periods (about 200 milliseconds). Under strict conditions, assuming ten times the power of one converter over another, Equation (17) will be satisfied in the twelfth period. It should be noted again that reactive power sharing could have a positive or negative effect on the convergence speed of active power sharing in the microgrids with complex impedances.

\section{Simulation RESUltS}

To evaluate the performance of the proposed algorithm, the microgrid benchmark in Fig. 2 is simulated in MATLAB/Simulink software with the parameters in Table II. In this structure, two DGs are integrated into the microgrid with complex impedance of lines to feed the common and local loads. Simulation studies are conducted for three different cases. In the first two cases, the single-bus structure and in the third case, the multi-bus structure is considered.

Case 1: Single-bus structure with complex impedance of lines

Initially, as the simplest case, the microgrid is in single-bus structure, and a common $R L$ load $(20 \Omega-5 \mathrm{mH})$ is fed by two inverters. As shown in Table II, the feeder impedance of the first inverter is complex and the feeder impedance of the second inverter is considered as pure resistive. The waveforms of the active and reactive power with and without the proposed controller are demonstrated in Fig. 6(a)-(b). The conventional droop scheme with virtual impedance in [22] is also compared with these waveforms. In the steady state, the power sharing without controller exhibits poor performance. In fact, without the proposed controller, the active power of $\mathrm{DG}_{2}$ is greater than $D_{1}$, but $D_{2}$ produces less reactive power, which shows unfair power sharing. Also, the active power is not shared properly in the conventional droop scheme due to the large and complex impedances. However, the proposed method achieves appropriate values for active and reactive power in less than 0.2 (a)

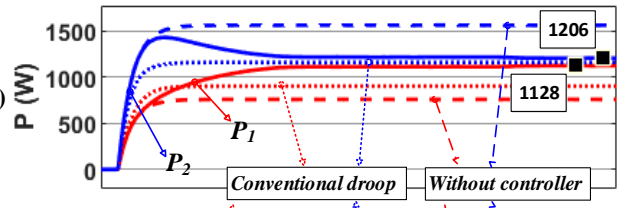

(b)

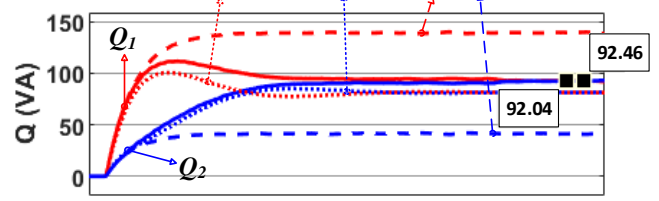

(c)

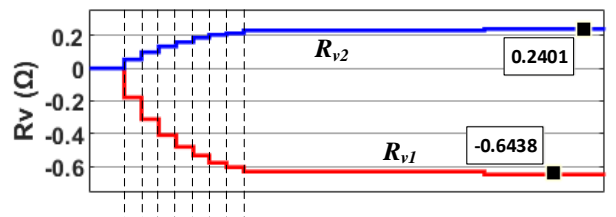

(d)

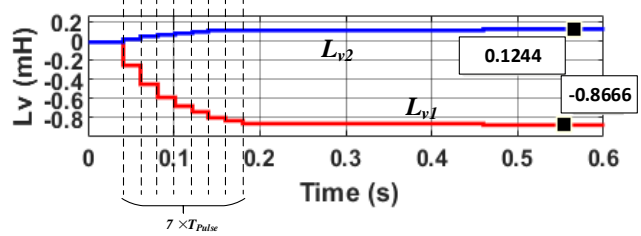

Fig. 6. Performance of the proposed algorithm under case 1. (a) Active power, (b) Reactive power, (c) Virtual resistance, (d) Virtual inductance.

(a)

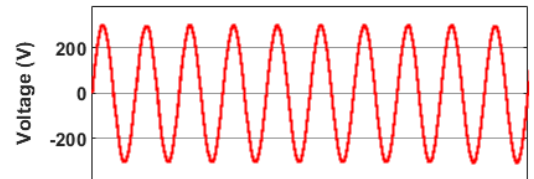

(b)

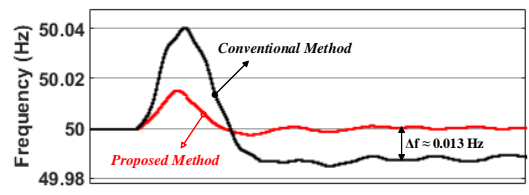

(c)

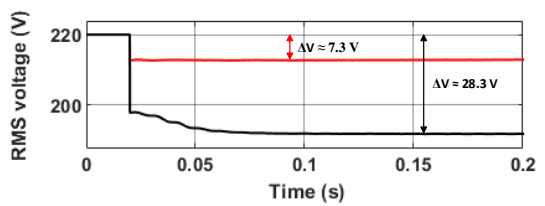

Fig. 7. Comparison of voltage for proposed and conventional method.

seconds and satisfies the algorithm in less than 0.5 seconds. The active and reactive power sharing errors are $6.7 \%$ and $0.5 \%$, respectively. It should be noted that the power sharing error is due to the algorithm constraints that can change the accuracy and dynamics of the method. Fig. 6(c)-(d) illustrates the values of virtual resistance and inductance, which surprisingly compensated for the difference in equivalent impedances. As can be seen, virtual impedances are updated every 20 milliseconds to the point where the power-sharing requirement is met. In this method, virtual impedance values are much lower than traditional droop methods, and negative virtual impedance can be used without the system information. The bus voltage and frequency and voltage deviations are also presented in Fig. 7. The proposed method is compared with the conventional droop method, which shows the superiority of the proposed method. The impedance-power method does not cause frequency deviation, unlike the conventional droop method. 
(a)

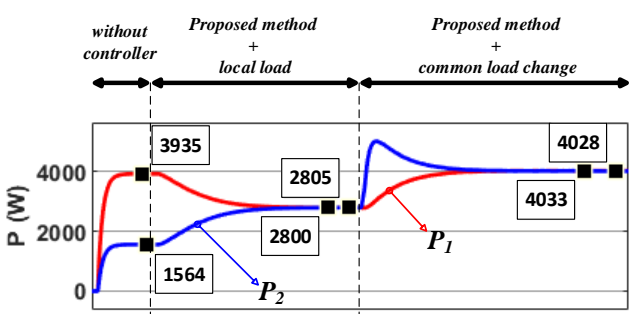

(b)

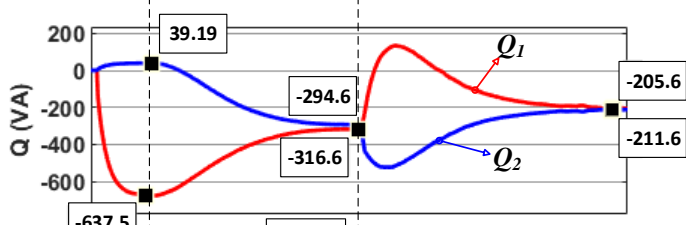

(c)

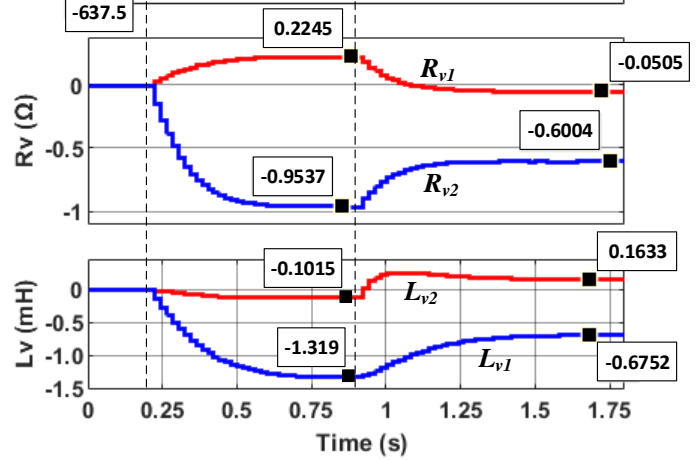

Fig. 8. Performance of the proposed algorithm under case 2. (a) Active power, (b) Reactive power, (c) Virtual resistance, (d) Virtual inductance.

(a)

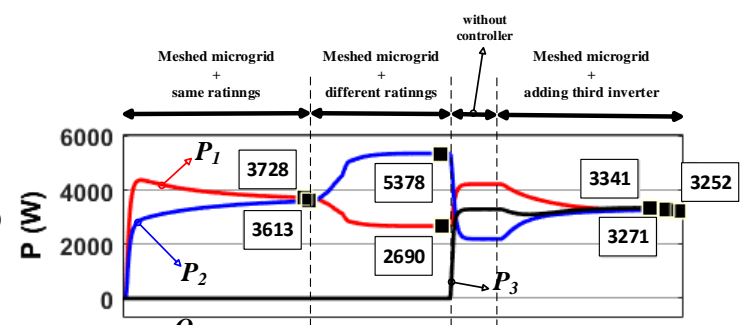

(b)

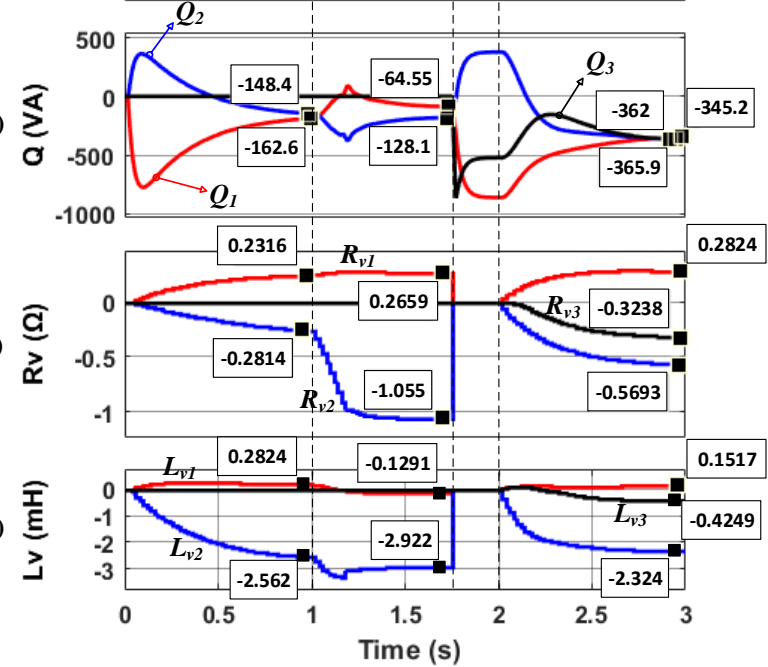

Fig. 9. Performance of the proposed algorithm under case 3. (a) Active power, (b) Reactive power, (c) Virtual resistance, (d) Virtual inductance.

Besides, the voltage magnitude deviation is much less than the conventional method due to smaller and negative virtual impedances. However, it should be noted that the voltage fluctuates within the standard range due to other considerations such as load changes. The bus voltage variations can be considered as the secondary virtual impedance for both inverters. The impact of this impedance on the virtual impedance variations obtained from the proposed algorithm (Equations (12) and (13)) is very small and negligible. Therefore, bus voltage information in the proposed method can be considered almost constant. In other words, in these equations, only a reference is needed to calculate the virtual impedance variations of the parallel inverters. Therefore, the dependence of the method on bus voltage will be solved by an initial sampling of the voltage, which is also necessary for synchronization, and the algorithm does not add complexity to the system.

\section{Case 2: Local and common load changes}

In this case, the non-linear local load is added at the output of the first inverter to check the performance of the proposed method in the presence of local loads. The non-linear load consists of a resistor $(2 \Omega)$ in series with a diode rectifier feeding a parallel R-C circuit $\left(\mathrm{R}_{\mathrm{N}}=20 \Omega, \mathrm{C}_{\mathrm{N}}=680 \mathrm{uF}\right)$. The rest of the system structure is similar to the previous case. The performance of the microgrid in different states of this case is shown in Fig. 8. Initially, the system is started without any controller for power sharing. Because of the microgrid structure, the active power of $\mathrm{DG}_{1}$ is about three times the second one, and while $\mathrm{DG}_{1}$ consumes significant reactive power, the second unit is the reactive power generator. Then, the proposed control method is applied in $t=0.2 \mathrm{~s}$, which uses bus voltage information at startup for the proposed method. As can be seen, the exact power sharing is achieved in less than 0.6 seconds. In fact, the proposed control method adjusts the virtual impedances so that local and common loads are shared proportionally between the inverters. In the last step in $t=0.9$ $s$, the common linear load (dynamic load) becomes double. Once a sudden load change is occurring, the proposed algorithm functions to compensate for the power mismatch automatically. At the beginning of this step, the injected power of the first inverter will increase immediately but the proposed controller is able to coordinate active and reactive power among the DG units. As a result, the proposed control algorithm achieves accurate power sharing in both active and reactive power under any load condition.

Case 3: Multi-bus microgrid with non-identical inverters

In this case, the performance of the proposed control method is investigated in the multi-bus structure with complex impedance of lines. For this purpose, the common bus has been removed and replaced by impedance $Z_{1,2}$ instead. As the last simulation study, three different states are examined. Fig. 9 shows the performance when DG units have the same and different power ratings, respectively. During state one $(0<\mathrm{t}<$ $1)$, the multi-bus microgrid is operated with the same power rating of DG units. As can be seen, the proposed method shares both active and reactive power efficiently regardless of network structure and complex impedance of lines. The DG units inject $3728 \mathrm{~W}$ and $3613 \mathrm{~W}$ and consume 162.6 Var and 148.4 Var, respectively. At the end, it is assumed that the nominal power of $\mathrm{DG}_{1}$ is reduced by half in $t=1 \mathrm{~s}$. By changing the droop gain, the virtual impedances are updated to reset the power sharing 
TABLE III

COMPARISON OF DIFFERENT CONTROL METHODS PERFORMANCE

\begin{tabular}{|c|c|c|c|c|c|}
\hline FEATURE & $\begin{array}{l}\text { VIRTUAL IMPEDANCE } \\
\text { CONTROLLER [18] }\end{array}$ & $\begin{array}{l}\text { LINEAR IMPEDANCE- } \\
\text { POWER DROOP [17] }\end{array}$ & $\begin{array}{c}\text { CONSENSUS } \\
\text { ALGORITHM [19], [20] }\end{array}$ & $\begin{array}{l}\text { CONTROL OF INHERENT } \\
\text { IMPEDANCE [22] }\end{array}$ & PROPOSED METHOD \\
\hline $\begin{array}{l}\text { CAPABILITY OF POWER SHARING IN } \\
\text { MULTI-BUS STRUCTURE }\end{array}$ & YES & No & YES & No & YES \\
\hline POWER SHARING ACCURACY & GoOD & EXCELLENT & EXCELLENT & FAIR & EXCELLENT \\
\hline COMPUTATIONAL AND COMPLEXITY & MEDIUM & Low & HIGH & HIGH & MEDIUM \\
\hline FREQUENCY DEVIATION & HIGH & Low & HIGH & HIGH & Low \\
\hline VOLTAGE REGULATION & FAIR & GoOD & POOR & GoOD & GoOD \\
\hline VIRTUAL IMPEDANCE & LARGE & MEDIUM & LARGE & Low & MEDIUM \\
\hline THD & $\mathrm{HIGH}$ & MEDIUM & $\mathrm{HIGH}$ & Low & MEDIUM \\
\hline LIMITATION & $\begin{array}{l}\text { EXACT RECOGNITION OF } \\
\text { STRUCTURE }\end{array}$ & SINGLE-BUS, LBC & COMPLEXITY, LBC & $\begin{array}{l}\text { SINGLE-BUS, LOW LINE } \\
\text { IMPEDANCES }\end{array}$ & $\begin{array}{c}\text { LOW BANDWIDTH } \\
\text { COMMUNICATION (LBC) }\end{array}$ \\
\hline
\end{tabular}

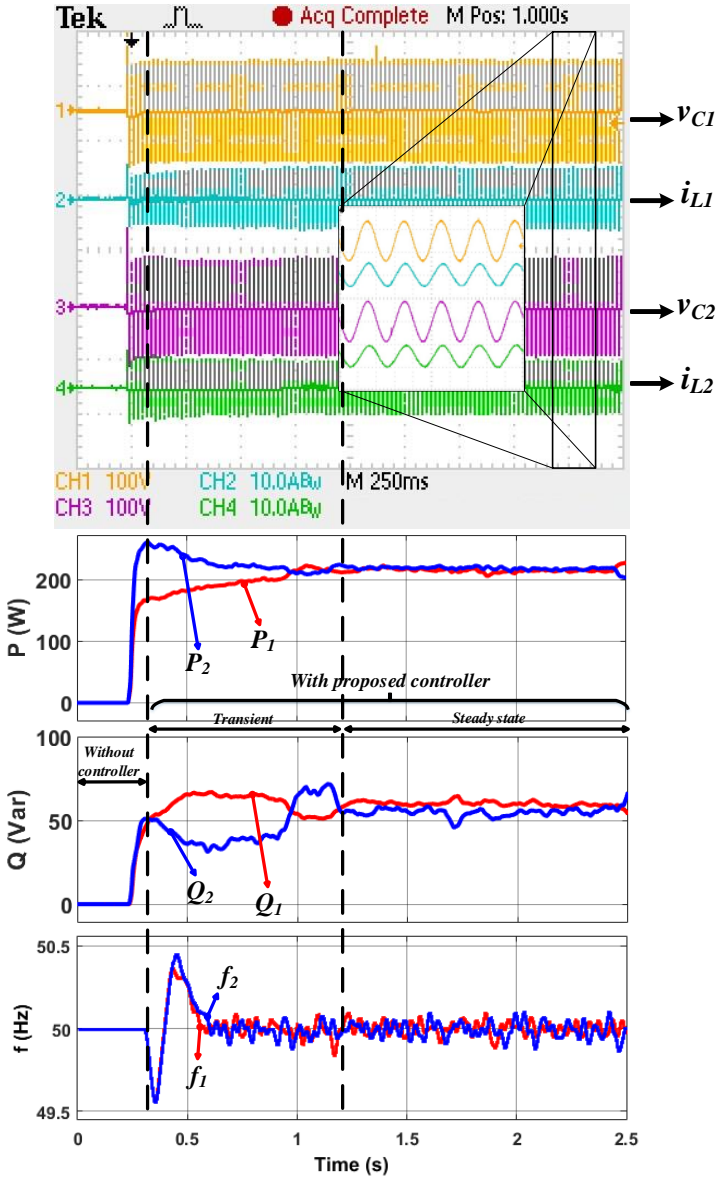

Fig. 10. Experimental power sharing performance of the proposed controller method.

based on the nominal power of the inverters. It can be seen that in spite of different ratings of inverters, the proposed method still works well. The active and reactive power sharing errors is $0.04 \%$ and $0.78 \%$, respectively. Finally, in the last step at $t=$ $1.75 s$, another inverter with a $R L$ line impedance $(1.5 \Omega-1.5$ $\mathrm{mH}$ ) and a non-linear local load is added to the output of the second inverter. In this state, at $t=2 \mathrm{~s}$, the proposed algorithm is applied again and by adjusting the proper virtual impedances, all inverters participate fairly in power generation. In other words, the proposed control method is suitable for microgrids even with more than two inverters and has the plug and play feature. In result, even when the microgrid is changed from a single-bus to a multi-bus form, accurate power sharing is achieved. A rough comparison between the proposed algorithm and some control methods is presented in Table III.

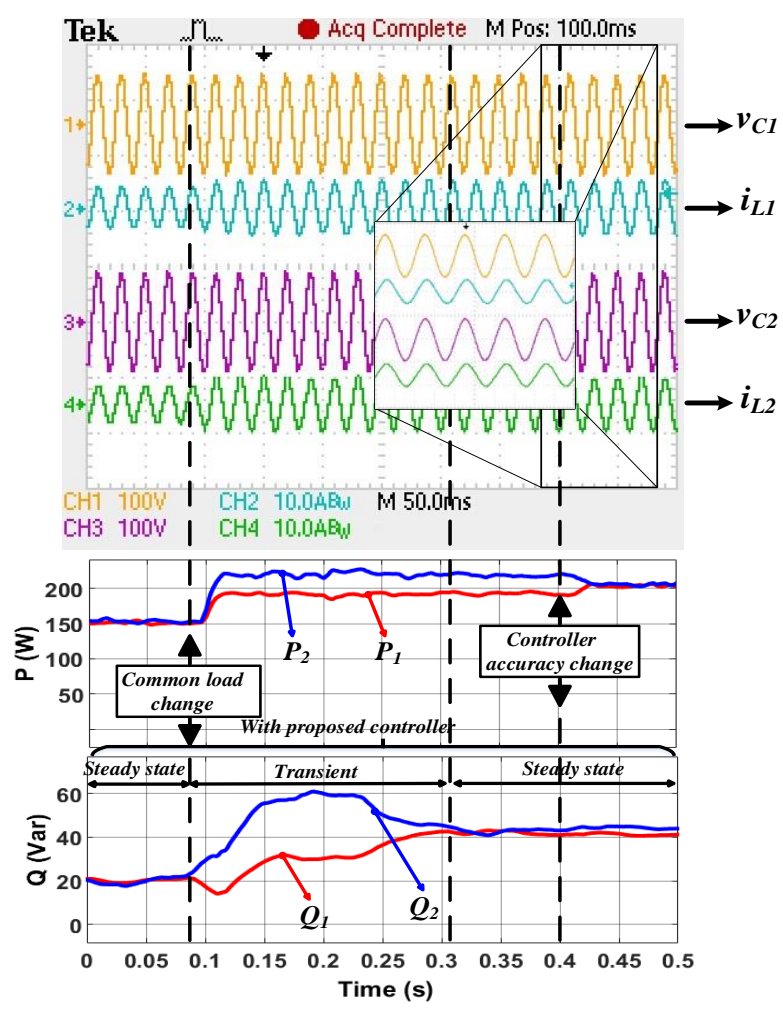

Fig. 11. Performance of the proposed control method in presence of common load change.

\section{EXPERIMENTAL VERIFICATION}

In this section, a laboratory-scale multi-bus microgrid is used to evaluate the feasibility of the proposed control method to achieve active and reactive power sharing. The hardware implementation is controlled by using a TMS320F28335 digital signal processor (Texas Instruments). The experimental system parameters are also listed in Table II. Due to the limitation of laboratory equipment to test the higher voltages, practical results are presented in $70 \mathrm{Vrms}$. To demonstrate the method efficacy, three experiments are analyzed.

In the first experiment, the performance of the proposed control method is investigated when applied to the system. The voltage and current waveforms of each inverter and their output power are shown in Fig. 10. The active power, reactive power and frequency are obtained indirectly from the voltage and current waveforms. Initially, microgrid has been launched without any controller for power sharing. As can be seen, the 
TABLE IV

POWER SHARING ERROR AND CONVERGENCE TIME OF THE PROPOSED ALGORITHM FOR DIFFERENT TESTS

\begin{tabular}{|c|c|c|c|c|c|c|c|c|c|}
\hline \multirow{3}{*}{ Test } & \multicolumn{6}{|c|}{ Simulation } & \multicolumn{3}{|c|}{ Experimental } \\
\hline & \multirow{2}{*}{ Case 1} & \multicolumn{2}{|c|}{ Case 2} & \multicolumn{3}{|c|}{ Case 3} & \multirow{2}{*}{ Case 1} & \multirow{2}{*}{ Case 2} & \multirow{2}{*}{ Case 3} \\
\hline & & Step 1 & Step 2 & Step 1 & Step 2 & Step 3 & & & \\
\hline $\begin{array}{c}\text { Active power } \\
\text { sharing error }(\%)\end{array}$ & 6.68 & 0.18 & 0.12 & 3.13 & 0.04 & $<2.70$ & $<2$ & $<10$ & $<2$ \\
\hline $\begin{array}{c}\text { Reactive power } \\
\text { sharing error }(\%)\end{array}$ & 0.45 & 7.20 & 2.88 & 9.13 & 0.78 & $<5.82$ & $<10$ & $<5$ & $<5$ \\
\hline $\begin{array}{c}\text { Convergence time } \\
(\mathrm{ms})\end{array}$ & 140 & 480 & 680 & 920 & 560 & 840 & 960 & 340 & 460 \\
\hline
\end{tabular}

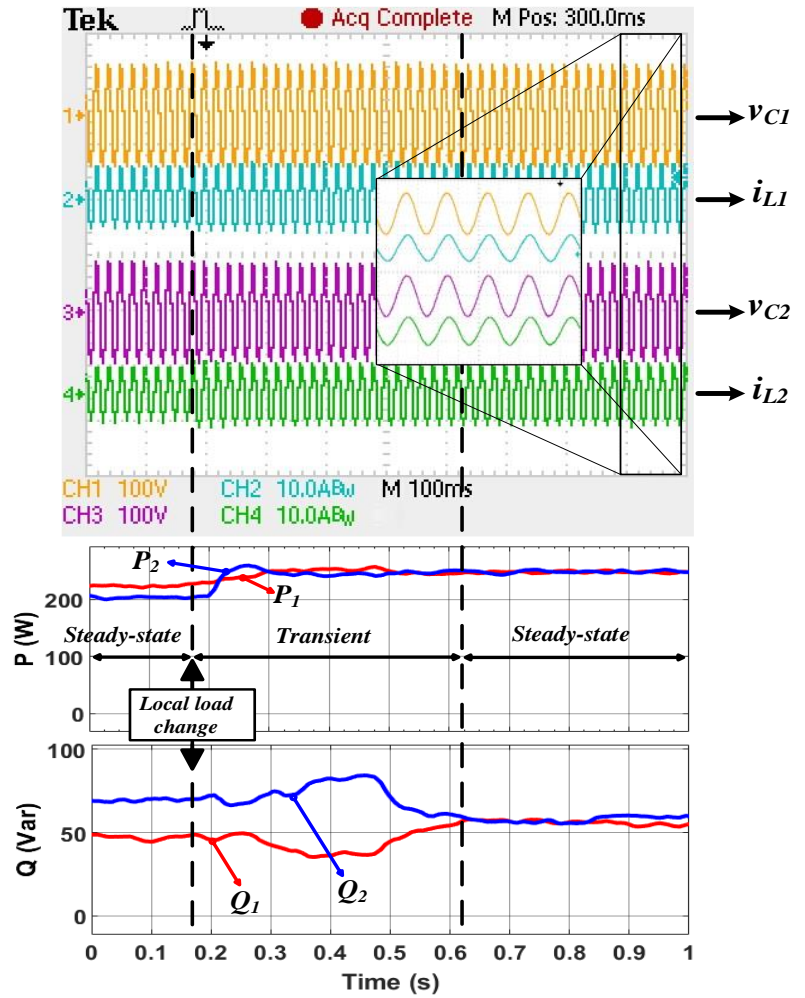

Fig. 12. Performance of the proposed control method in presence of local load.

active and reactive power between the two DG units is not the same. At $t=0.3 \mathrm{~s}$, the proposed controller is activated, which after about 0.9 seconds, the system reaches steady state and active and reactive power are shared equally. The zoomed waveforms of the voltage and current in steady state are also shown in Fig. 10, which are identical for both DGs. Also, the frequency waveforms for both inverters show their nondeviation from the reference values similar to the simulation result.

In the next experiment, the transient performance of the proposed method is studied when the common load is changed suddenly. Fig. 11 shows the waveforms associated with this case. In this test, the resistance of common load 1 changes from $24 \Omega$ to $12 \Omega$ at $t=0.09 \mathrm{~s}$. With the violation of the constraint of reactive power sharing, the proposed controller is activated and after about 0.2 seconds, the desired results are reached. Although the accurate reactive power sharing can be achieved, an inappropriate difference exists in the active power sharing due to the precision constraint of the algorithm. At $t=0.4 \mathrm{~s}$, the accuracy of the proposed method is improved with the change in the impedance-power droop coefficient, which the result can be well observed in active power sharing.

Finally, the performance of the proposed control method in the presence of local load is examined and the results are depicted in Fig. 12. In this experiment, the local load of $48 \mathrm{ohms}$ is added to the output of the second inverter. At $t=0.18 \mathrm{~s}$, the proposed controller is activated and the system achieves accurate power sharing after about 0.45 seconds by adjusting the virtual impedances.

For the sake of more clarity, the power sharing error and convergence time of the proposed algorithm are presented in Table IV for all simulation and experimental tests. As can be seen, the power sharing error is less than $10 \%$ for all tests, which satisfy the power-sharing condition. Also, the convergence time is within a satisfactory range, which shows the acceptable performance of the proposed method.

\section{CONCLUSION}

The active and reactive power sharing in multi-bus microgrids with complex impedance of lines have significant challenges because the network structure is variable, the specific feeder impedance cannot be defined and there is a coupling between active and reactive powers. In this paper, a simple control algorithm based on complex virtual impedance was presented to obtain accurate power sharing between DG units. The proposed control algorithm uses a modified droop method, called extended impedance-power droop, to systematically adjust the equivalent inverter impedance. The resistive and inductive components of the virtual impedance was adaptively obtained by using nonlinear extended droop. This method did not require the identification of the system parameters, high bandwidth communication links, and the use of traditional droop method. In addition, with the proposed control scheme, the inherent frequency deviation of droop methods is eliminated. Finally, the simulation and practical results confirmed the correct operation under different operating scenarios. This method always provides accurate power sharing for both active and reactive power under different load conditions and microgrid configurations.

\section{REFERENCES}

T. Strasser et al., "A Review of Architectures and Concepts for Intelligence in Future Electric Energy Systems," IEEE Trans. Ind. Electron., vol. 62, no. 4, pp. 2424-2438, Apr. 2015.

[2] K. Thirugnanam, S. K. Kerk, C. Yuen, N. Liu, and M. Zhang, "Energy Management for Renewable Microgrid in Reducing Diesel 
Generators Usage With Multiple Types of Battery," IEEE Trans. Ind. Electron., vol. 65, no. 8, pp. 6772-6786, Aug. 2018.

[3] L. Lin, H. Ma, and Z. Bai, "An Improved Proportional Load-Sharing Strategy for Meshed Parallel Inverters System With Complex Impedances," IEEE Trans. Power Electron., vol. 32, no. 9, pp. 73387351, Sep. 2017.

[4] L. Zhou et al., "Harmonic Voltage Distortion Damping Method for Parallel-connected LCL-type Inverters in Islanded Operation," IEEE Trans. Ind. Electron., vol. PP, no. c, pp. 1-1, 2018.

[5] A. Ovalle, G. Ramos, S. Bacha, A. Hably, and A. Rumeau, "Decentralized Control of Voltage Source Converters in Microgrids Based on the Application of Instantaneous Power Theory," IEEE Trans. Ind. Electron., vol. 62, no. 2, pp. 1152-1162, Feb. 2015.

[6] M. J. Hossain, M. A. Mahmud, F. Milano, S. Bacha, and A. Hably, "Design of Robust Distributed Control for Interconnected Microgrids," IEEE Trans. Smart Grid, vol. 7, no. 6, pp. 2724-2735, Nov. 2016.

[7] E. Chiodo and D. Lauria, "Some Basic Properties of the Failure Rate of Redundant Reliability Systems in Industrial Electronics Applications," IEEE Trans. Ind. Electron., vol. 62, no. 8, pp. 50555062, Aug. 2015.

[8] M. Yazdanian and A. Mehrizi-Sani, "Distributed Control Techniques in Microgrids," IEEE Trans. Smart Grid, vol. 5, no. 6, pp. 29012909, Nov. 2014.

[9] M. Ramezani, S. Li, and Y. Sun, "Combining droop and direct current vector control for control of parallel inverters in microgrid," IET Renew. Power Gener., vol. 11, no. 1, pp. 107-114, Jan. 2017.

[10] H. Moussa, A. Shahin, J.-P. Martin, S. Pierfederici, and N. Moubayed, "Optimal Angle Droop for Power Sharing Enhancement With Stability Improvement in Islanded Microgrids," IEEE Trans. Smart Grid, vol. 9, no. 5, pp. 5014-5026, Sep. 2018.

[11] A. Trivedi and M. Singh, "\$L_ $\{1\} \$$ Adaptive Droop Control for AC Microgrid With Small Mesh Network," IEEE Trans. Ind. Electron., vol. 65, no. 6, pp. 4781-4789, Jun. 2018.

[12] J. C. Vasquez, J. M. Guerrero, A. Luna, P. Rodríguez, and R. Teodorescu, "Adaptive droop control applied to voltage-source inverters operating in grid-connected and islanded modes," IEEE Trans. Ind. Electron., vol. 56, no. 10, pp. 4088-4096, 2009.

[13] J. He, Y. Pan, B. Liang, and C. Wang, "A Simple Decentralized Islanding Microgrid Power Sharing Method Without Using Droop Control," IEEE Trans. Smart Grid, vol. 9, no. 6, pp. 6128-6139, 2018.

[14] T. V. Hoang and H. H. Lee, "Accurate Power Sharing with Harmonic Power for Islanded Multibus Microgrids," IEEE J. Emerg. Sel. Top. Power Electron., vol. 7, no. 2, pp. 1286-1299, 2019.

[15] J. He, Y. W. Li, J. M. Guerrero, F. Blaabjerg, and J. C. Vasquez, “An islanding Microgrid power sharing approach using enhanced virtual impedance control scheme," IEEE Trans. Power Electron., vol. 28, no. 11, pp. 5272-5282, 2013.

[16] Y. Zhu, F. Zhuo, F. Wang, B. Liu, and Y. Zhao, "A Wireless Load Sharing Strategy for Islanded Microgrid Based on Feeder Current Sensing," IEEE Trans. Power Electron., vol. 30, no. 12, pp. 67066719, 2015.

[17] R. Razi, H. Iman-Eini, and M. Hamzeh, "An Impedance-Power Droop Method for Accurate Power Sharing in Islanded Resistive Microgrids," IEEE J. Emerg. Sel. Top. Power Electron., pp. 1-1, 2019.

[18] Y. Zhu, F. Zhuo, F. Wang, B. Liu, R. Gou, and Y. Zhao, "A virtual impedance optimization method for reactive power sharing in networked microgrid," IEEE Trans. Power Electron., vol. 31, no. 4, pp. 2890-2904, 2016.

[19] H. Zhang, R. Han, J. Zhou, S. Kim, and Q. Sun, "Consensus-Based Distributed Control for Accurate Reactive, Harmonic, and Imbalance Power Sharing in Microgrids," IEEE Trans. Smart Grid, vol. 9, no. 4, pp. 2453-2467, 2016.

[20] H. Zhang, S. Kim, Q. Sun, and J. Zhou, "Distributed Adaptive Virtual Impedance Control for Accurate Reactive Power Sharing Based on Consensus Control in Microgrids," IEEE Trans. Smart Grid, vol. 8, no. 4, pp. 1749-1761, Jul. 2017.

[21] H. Mahmood, D. Michaelson, and J. Jiang, "Accurate reactive power sharing in an islanded microgrid using adaptive virtual impedances," IEEE Trans. Power Electron., vol. 30, no. 3, pp. 1605-1617, 2015.

[22] P. Sreekumar and V. Khadkikar, "Direct control of the inverter impedance to achieve controllable harmonic sharing in the Islanded Microgrid," IEEE Trans. Ind. Electron., vol. 64, no. 1, pp. 827-837, 2017.

[23] T. V. Hoang and H. H. Lee, "An Adaptive Virtual Impedance Control Scheme to Eliminate the Reactive-Power-Sharing Errors in an Islanding Meshed Microgrid," IEEE J. Emerg. Sel. Top. Power Electron., vol. 6777, no. c, 2017.

[24] O. V. Kulkarni, S. Doolla, and B. G. Fernandes, "Simple Controller Configuration for Decentralized Parallel Operation of Inverters," IEEE Trans. Power Electron., vol. 34, no. 2, pp. 1356-1369, 2019.

[25] R. Razi, M.-S. Karbasforooshan, and M. Monfared, "Multi-loop control of UPS inverter with a plug-in odd-harmonic repetitive controller," ISA Trans., vol. 67, pp. 496-506, 2017. 